\title{
Localization of Cdc42 in the Rat Seminiferous Epithelium and Immortalized Sertoli Cell Lines
}

\author{
John Rollinson, Purnima Jani, and Clarke F. Millette
}

Department of Cell and Developmental Biology and Anatomy, University of South Carolina School of Medicine, 6439 Garners Ferry Road, SC, 29209, USA

Sertoli cells, the epithelial component of the mammalian seminiferous epithelium, undergo dramatic and repetitive rearrangements of their actin cytoskeleton as they progress through the various stages of the spermatogenic cycle. Co-ordination of Sertoli cell shape with respect to the closely associated cohort of differentiating germ cells is absolutely crucial to continuation of normal sperm cell production. Small GTPases of the Rho family control actin cytoskeletal rearrangements in many cells, including Sertoli cells. Recent data demonstrate involvement of Cdc42 in Sertoli cell-germ cell adhesive interactions [1]. However, the precise expression patterns and functions of Cdc42 in the mammalian testis remain under-investigated. Here, we describe the localization of the Rho family GTPase Cdc42 in the rat testis.

Western blot analysis demonstrates that antibodies specific for $\mathrm{Cdc} 42$ recognize protein expressed in both rat Sertoli cells and developing germ cells of the rat testis. RT-PCR and subsequent cloning studies confirm that this protein is identical to rat somatic cell $\mathrm{Cdc} 42$.

Immunofluorescent analysis demonstrates that while some Cdc42 is localized in a diffuse cytoplasmic distribution, a peri-nuclear concentration of $\mathrm{Cdc} 42$ predominates in primary Sertoli cell isolates from immature (post-partum day 16-17) rats, and in the immortalized Sertoli cell lines, ASC-17D and 15P1. Cdc42 positioning does not coincide with filamentous actin, mitochondria, lysosomes, or microtubules. However, expression of Cdc42 is clearly dependent upon the stage of the spermatogenic cycle. Late condensing spermatids express increased levels of Cdc42. Sertoli cells express $\mathrm{Cdc} 42$ protein throughout the spermatogenic cycle. Analysis of rat testes during initial development of spermatogenesis suggest that Cdc42 is involved in actin rearrangements of early prepachytene spermatocytes, but is less important in developing pachytene or diplotene cells.

Physiological functions of $\mathrm{Cdc} 42$ in the seminiferous tubule remain to be elucidated. An interaction with Cdc42 and proteins governing specific Sertoli cell-germ cell adhesive connections has been demonstrated recently [1], but a detailed understanding of Rho-family GTPases in the mammalian testis remains elusive. Specific triggering of the Sertoli cell surface cell adhesion molecule L-selectin activates the Rho-family GTPase Rac-1, but does not significantly affect Cdc42. Similar results derive from experiments activating cells using LPA.

Therefore, data presented here suggest that Cdc42 in the seminiferous epithelium is indeed involved in Sertoli cell - germ cell interactions, but that this involvement is complicated and dependent upon the precise stage of the spermatogenic cycle. Molecular intracellular interactions between RhoA, Rac1 and Cdc42 need to be examined during in more detail during mammalian spermatogenesis.

[1] W. Y. Lui et. al. J. Cell Physiol. 202(1) (2005) 49.

[2] Supported by HD-37280 CFM 

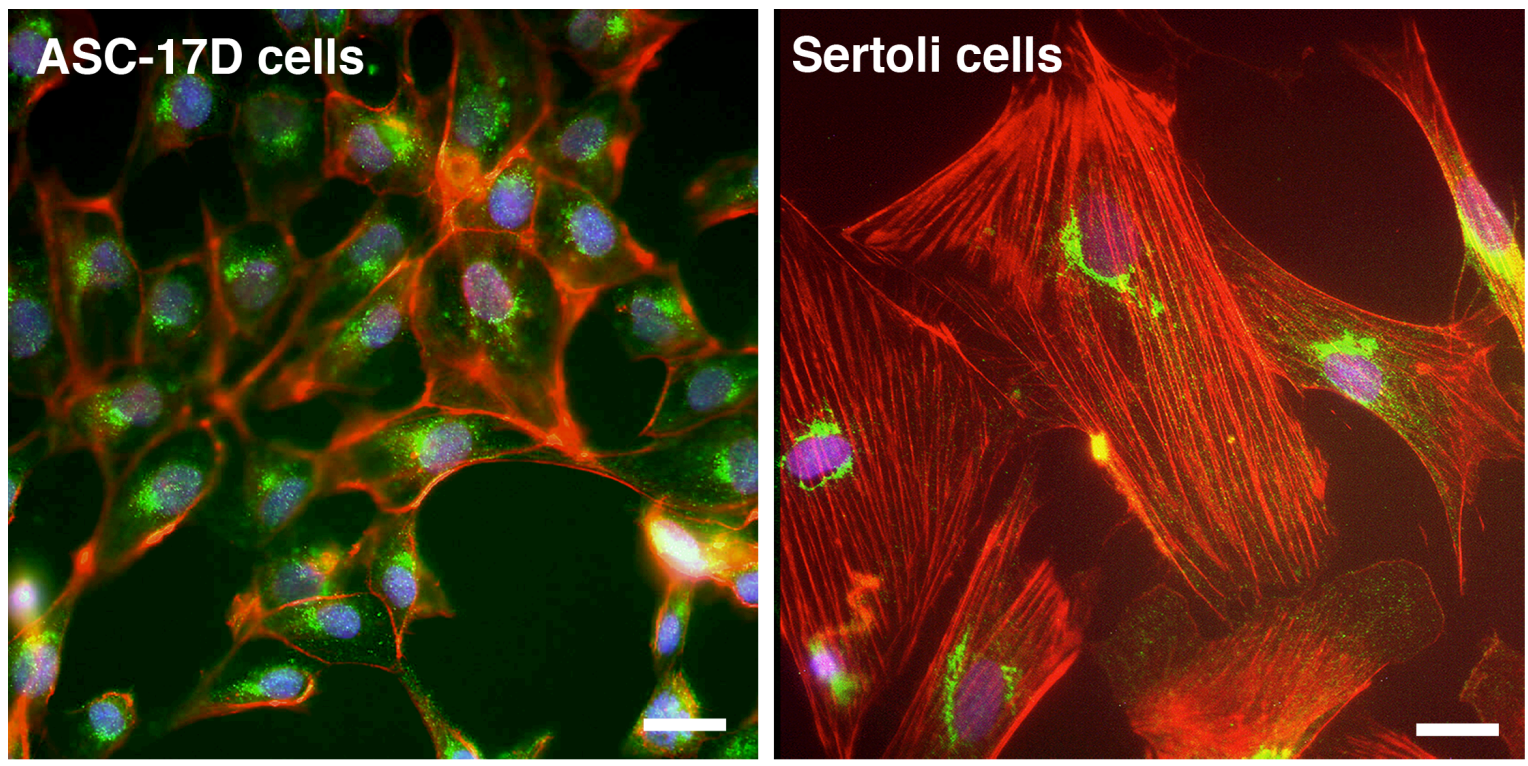

Figure 1: ASC-17D cells and Sertoli cells immunostained for Cdc42 (green), F-actin (red) and DNA (blue). Cdc42 is concentrated in a distinctive perinuclear localization. Scale bar $=15 \mu \mathrm{m}$.

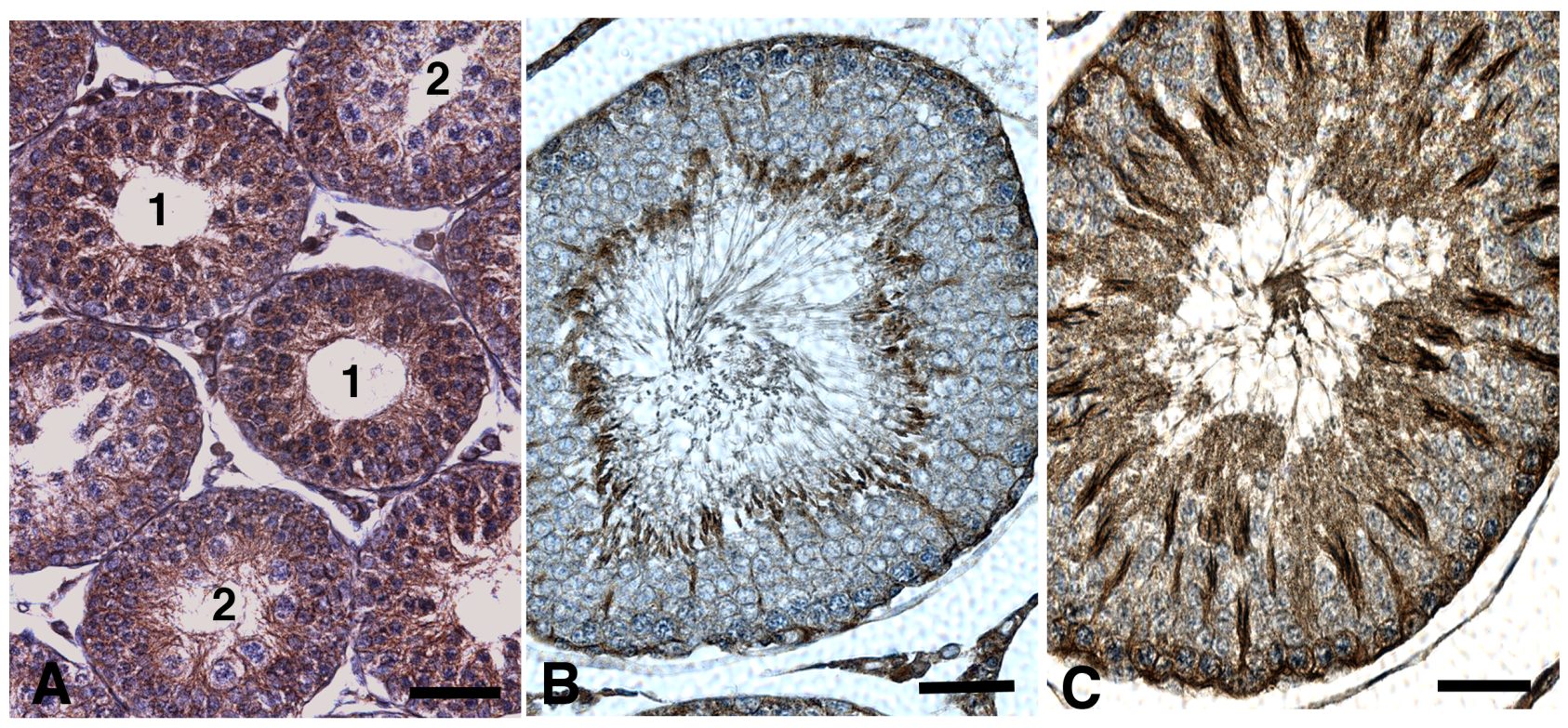

Figure 2: Immunohistochemical staining of immature day 16 rat testis $(A)$ and adult rat testis $(B)$ and $(C)$ for Cdc42. Note the differential staining of cells in immature seminiferous tubules at varying stages of spermatogenic development. Compare tubules labeled "1" and "2". Panel B shows a seminiferous tubule at stage 7 of the spermatogenic cycle, while panel $C$ shows in a tubule in the same tissue section at stage 13. Clearly, Cdc42 expression varies according to the precise interactions between developing germ cells and their surrounding epithelial Sertoli cells. Scale bars $=50 \mu \mathrm{m}$. 